\title{
Apparent Treatment-Resistant Hypertension and Chronic Kidney Disease: Another Cardiovascular-Renal Syndrome?
}

\author{
Sreekanth Vemulapalli, Crystal C. Tyson, and Laura P. Svetkey
}

\begin{abstract}
To identify patients at increased risk of cardiovascular (CV) outcomes, apparent treatment-resistant hypertension (aTRH) is defined as having a blood pressure above goal despite the use of 3 or more antihypertensive therapies of different classes at maximally tolerated doses, ideally including a diuretic. Recent epidemiologic studies in selected populations estimated the prevalence of aTRH as $10 \%$ to $15 \%$ among patients with hypertension and that aTRH is associated with elevated risk of CV and renal outcomes. Additionally, aTRH and CKD are associated. Although the pathogenesis of aTRH is multifactorial, the kidney is believed to play a significant role. Increased volume expansion, aldosterone concentration, mineralocorticoid receptor activity, arterial stiffness, and sympathetic nervous system activity are central to the pathogenesis of aTRH and are targets of therapies. Although diuretics form the basis of therapy in aTRH, pathophysiologic and clinical data suggest an important role for aldosterone antagonism. Interventional techniques, such as renal denervation and carotid baroreceptor activation, modulate the sympathetic nervous system and are currently in phase III trials for the treatment of aTRH. These technologies are as yet unproven and have not been investigated in relationship to CV outcomes or in patients with CKD.
\end{abstract}

(C) 2014 by the National Kidney Foundation, Inc. All rights reserved.

Key Words: Resistant hypertension, Chronic kidney disease, Aldosterone, Diuretic, Cardiovascular outcomes

\section{Introduction}

Hypertension is the number one attributable risk factor for cardiovascular $(\mathrm{CV})$ disease and is responsible for over half of the estimated 17 million deaths per year resulting from CV disease worldwide. ${ }^{1}$ Every $20 / 10 \mathrm{~mm} \mathrm{Hg}$ increase in blood pressure (BP) correlates with a doubling of $\mathrm{CV}$ mortality. ${ }^{2}$ Beyond mortality, hypertension is a significant risk factor for $\mathrm{CV}$ morbidity, including stroke, myocardial infarction, heart failure, and renal failure. ${ }^{3}$ These events occur at higher rates in individuals with apparent treatment-resistant hypertension (aTRH) ${ }^{4}$ and in individuals with chronic kidney disease (CKD). aTRH is particularly prevalent in patients with CKD. ${ }^{5}$ This review will discuss the role of clinical and subclinical kidney disease in the epidemiology, pathogenesis, and treatment of aTRH.

\section{Definition of Resistant Hypertension}

To identify a subset of patients at increased CV risk who may benefit from special therapeutic considerations, the American Heart Association (AHA) defined resistant hypertension in a 2008 scientific statement as BP that remains above goal despite the use of 3 optimally dosed antihypertensive agents of different classes. ${ }^{6}$ By these terms, resistant hypertension includes patients at goal BP using 4 or more antihypertensive agents. Implicit in this definition is the exclusion of causes of pseudoresistant hypertension, including white-coat hypertension, medication nonadherence, and improper BP measurement technique. To effectively exclude causes of pseudoresistant hypertension, current guidelines recommend obtaining either home BPs or, if available, ambulatory BPs. ${ }^{6,7}$ Furthermore, patient compliance with antihypertensive therapy should be assessed by medication reconciliation and questioning family members.?

\section{Definition of aTRH}

aTRH has been coined to comprise patients meeting the AHA definition of resistant hypertension in whom causes for pseudoresistance have not been thoroughly investigated. ${ }^{8}$ Given that the vast majority of studies on resistant hypertension in the literature do not completely address pseudoresistance, we will use the term aTRH for the rest of this review unless pseudoresistance has been specifically excluded.

\section{Epidemiology of aTRH}

The prevalence of resistant hypertension is ill-defined. The accuracy of past estimates is complicated by a number of factors, including lack of a uniform definition for hypertension resistance, presence of referral bias in observed clinical cohorts, and the likely inclusion of individuals with pseudoresistant hypertension in observational studies. More recently, large prospective cohort studies have investigated the incidence and prevalence of aTRH. Daugherty and colleagues ${ }^{9}$ examined the incidence of aTRH among patients in the combined Kaiser Northern California and Kaiser Colorado databases. Among 205,750 newly diagnosed hypertensive patients, $1.9 \%$ were found to have aTRH, defined as uncontrolled BP on 3 or more antihypertensive medications or controlled BP on 4 or more antihypertensive medications, plus at least $80 \%$ medication adherence at

From Division of Cardiology, Department of Medicine, Duke University Medical Center, Durham, NC; Division of Nephrology, Department of Medicine, Duke University Medical Center, Durham, NC.

Financial Disclosures: S.V. reports grant funding from Boston Scientific and speaker's fees from Medtronic. C.C.T. reports speaker's fees and grant funding from Medtronic and grant funding from the National Institutes of Health (grant 1U01-HL-096720). L.P.S. reports grant funding from the National Institutes of Health (grant 1U01-HL-096720) and speaker's fees and grant funding from Medtronic.

Address correspondence to Sreekanth Vemulapalli, $M D$, Division of Cardiology, Duke University Medical Center, Box 3126, Durham, NC 27710. E-mail: squster@gmail.com

(c) 2014 by the National Kidney Foundation, Inc. All rights reserved.

$1548-5595 / \$ 36.00$

http://dx.doi.org/10.1053/j.ackd.2014.08.006 
1.5 years of follow-up. Among those taking 3 medications or more for at least 1 month, the prevalence of aTRH was $16.2 \%$. ${ }^{9}$ Given that medication noncompliance is likely the most common cause of pseudoresistance, the study by Daugherty and colleagues represents the most careful estimate of aTRH to date.

Other large cohort studies have documented a similar but growing prevalence of aTRH. Using sequential National Health and Nutrition Examination Survey (NHANES) data, Egan and colleagues examined the prevalence of aTRH, defined as BP of $140 / 90 \mathrm{~mm} \mathrm{Hg}$ or more while taking 3 or more antihypertensive medications, irrespective of dose, adherence, and BP measurement artifacts. From 1988 to 1994 to 2005 to 2008, the prevalence of aTRH among patients with hypertension grew from $5.5 \%$ to $11.8 \% .{ }^{10}$ Similarly, in the International Reduction of Atherothrombosis for Continued Health (REACH) Registry of 67,888 patients with known atherosclerotic disease or atherosclerotic risk factors enrolled from 2003 to 2004, the prevalence of aTRH was $12.7 \%$ among hypertensive patients. ${ }^{11}$ Thus, aTRH appears to affect about $12 \%$ of hypertensive adults, an estimated 8 million individuals in the United States.

\section{CKD and aTRH}

Among patients with hypertension, CKD is a common comorbidity with a prevalence of $32 \%$ in those with diagnosed hypertension and $24 \%$ in those with undiagnosed hypertension. ${ }^{12}$ Among those with aTRH, prospective cohort studies demonstrate remarkable consistency in the prevalence of CKD. In the REACH Registry, the prevalence of CKD at baseline, defined as estimated glomerular filtration rate (eGFR) less than $60 \mathrm{~mL} / \mathrm{min} / 1.73 \mathrm{~m}^{2}$, was $40.1 \%$, whereas in 1999 to 2004 and 2005 to 2008 , NHANES data showed a prevalence of $36.1 \%$ and $37.8 \%$, respectively, among those with aTRH. Furthermore, Egan and colleagues reported an eGFR less than $60 \mathrm{~mL} / \mathrm{min} / 1.73 \mathrm{~m}^{2}$ and albumin-tocreatinine ratio (ACR) greater than $300 \mathrm{mg} / \mathrm{g}$ to be associated with an increased odds ratio for aTRH among patients with hypertension. ${ }^{10}$ Thus, among patients with aTRH, CKD is common and proteinuria is associated with increased risk for aTRH in patients with hypertension.

Conversely, those with CKD have increased risk of aTRH: an analysis from the Reasons for Geographic and Racial Differences in Stroke (REGARDS) cohort study demonstrated an increasing prevalence and risk of aTRH among patients with lower eGFR and higher ACR (Table 1). ${ }^{13}$ Among patients with CKD, an elevated ACR in the setting of normal eGFR was also associated with an increased prevalence of aTRH. Thus, aTRH and CKD are commonly comorbid. However, given that not all pa- tients with aTRH have CKD and not all patients with CKD have aTRH, it is likely that mechanisms indirectly related to or independent of the kidney contribute to the pathophysiology of aTRH.

\section{Proposed Pathophysiology of aTRH}

The pathophysiology of aTRH is multifactorial (Fig 1), and the degree to which culpable factors contribute to treatment resistance likely varies among individuals. The kidney is believed to play a significant role in the pathogenesis of aTRH. The interplay between volume expansion, aldosterone, mineralocorticoid receptor (MR) activity, the sympathetic nervous system (SNS), and arterial stiffness are highlighted subsequently.

\section{Volume Expansion}

The volume expansion that accompanies CKD contributes significantly to hypertension. Johnson and colleagues have suggested that primary subclinical renal microvascular disease leading to afferent arteriolopathy and tubulointerstitial disease may be responsible for the development of salt-sensitive hypertension. ${ }^{14}$ Progressive tubulointerstitial disease will eventually result in microalbuminuria before the development of clinically apparent impairment of glomerular filtration. Concurrent microvascular damage is thought to result in renal vasoconstriction and subsequent local generation of angiotensin II. The resulting increased vascular resistance, reduced rate of ultrafiltration, and decreased sodium excretion cause sodium retention, volume expansion, and hypertension (Fig 2). ${ }^{15}$ Additional inciting factors leading to subclinical microvascular injury include sympathetic overactivity, increased activity of the renin-angiotensin axis, decreased production of nitric oxide (NO), low potassium diet, ${ }^{16,17}$ and presence of long-standing hypertension itself. Furthermore, these insults may be potentiated by known genetic factors affecting renal sodium handling and renin-angiotensin activity. ${ }^{18,19}$

\section{Aldosterone and the Mineralocorticoid Receptor}

Although the central roles of renin, angiotensin, and angiotensin II in the pathophysiology of hypertension have been well appreciated, the role of aldosterone has recently received increased attention. Up to $20 \%$ of patients consecutively referred to a hypertension clinic for aTRH were diagnosed with primary hyperaldosteronism based on suppressed renin activity and high 24-hour urinary aldosterone excretion. ${ }^{20}$ Aldosterone is produced in the adrenal glands in response to local and systemic angiotensin II and serum potassium concentrations. It binds MRs found in the 
Table 1. Prevalence of RH Among Patients With CKD and Albuminuria

MultivariableAdjusted

\section{Characteristic}

Prevalence Ratio*

$\begin{array}{lcc}\text { eGFR, } \mathrm{mL} / \mathrm{min} & & \\ \geq 60(n=8876) & 1 \text { (ref) } & \\ 40-59(n=1166) & 1.25(1.11-1.41) & \\ <45(n=658) & 1.2(1.04-1.37) & \\ \mathrm{ACR}, \mathrm{mg} / \mathrm{g} & & \mathrm{eGFR} \geq 60 \mathrm{~mL} / \mathrm{min} \\ <10(n=6166) & 1 \text { (ref) } & 1 \text { (ref) } \\ 10-29(n=2580) & 1.54(1.39-1.71) & 1.59(1.41-1.79) \\ 30-299(n=1536) & 1.76(1.57-1.97) & 1.88(1.65-2.14) \\ \geq 300(n=418) & 2.44(2.12-2.81) & 2.73(2.25-3.31)\end{array}$

Abbreviations: ACR, albumin-to-creatinine ratio; eGFR, estimated glomerular filtration rate.

* Multivariable adjustment included age, race, sex, region of residence, education, income, physical activity, current smoking, alcohol use, statin use, waist circumference, diabetes, total cholesterol level, high-density lipoprotein cholesterol level, C-reactive protein level, and history of myocardial infarction or stroke.

Data from Tanner et al. ${ }^{13}$

nephron and cardiomyocytes, cardiac fibroblasts, and vascular smooth muscle cells (Fig 2). ${ }^{21,22}$ The widespread locations of the MR are thought to explain the pleiotropic effects of aldosterone, including salt reabsorption, cardiac fibrosis, and vascular hypertrophy and inflammation resulting in reduced $\mathrm{NO}$ availability. Vascular inflammation leads to vascular fibrosis, whereas NO reduction leads to endothelial dysfunction and vasoconstriction. These pathologic changes may be central to increased vascular stiffness evidenced by increased pulse wave velocity seen in hypertension. ${ }^{23}$ Importantly, elevated plasma aldosterone level is not the only mechanism by which increased MR activity occurs. In the setting of CKD, the small GTPase protein Rac-1 also increases MR activation, causing hypertension. ${ }^{22}$ Thus, MR-mediated hypertension is possible even in patients with normal aldosterone concentrations. Indeed, among 1688 nonhypertensive patients in the Framingham Heart Study, baseline plasma aldosterone levels predicted progression to hypertension, even among patients with normal aldosterone concentrations at baseline. ${ }^{24}$

\section{Volume Expansion, Aldosterone, and CKD}

Both animal and human data have confirmed the importance of aldosterone in the pathogenesis and progression of CKD. In states of chronic volume expansion, which is often observed in CKD, the relationship between extracellular volume and plasma aldosterone is

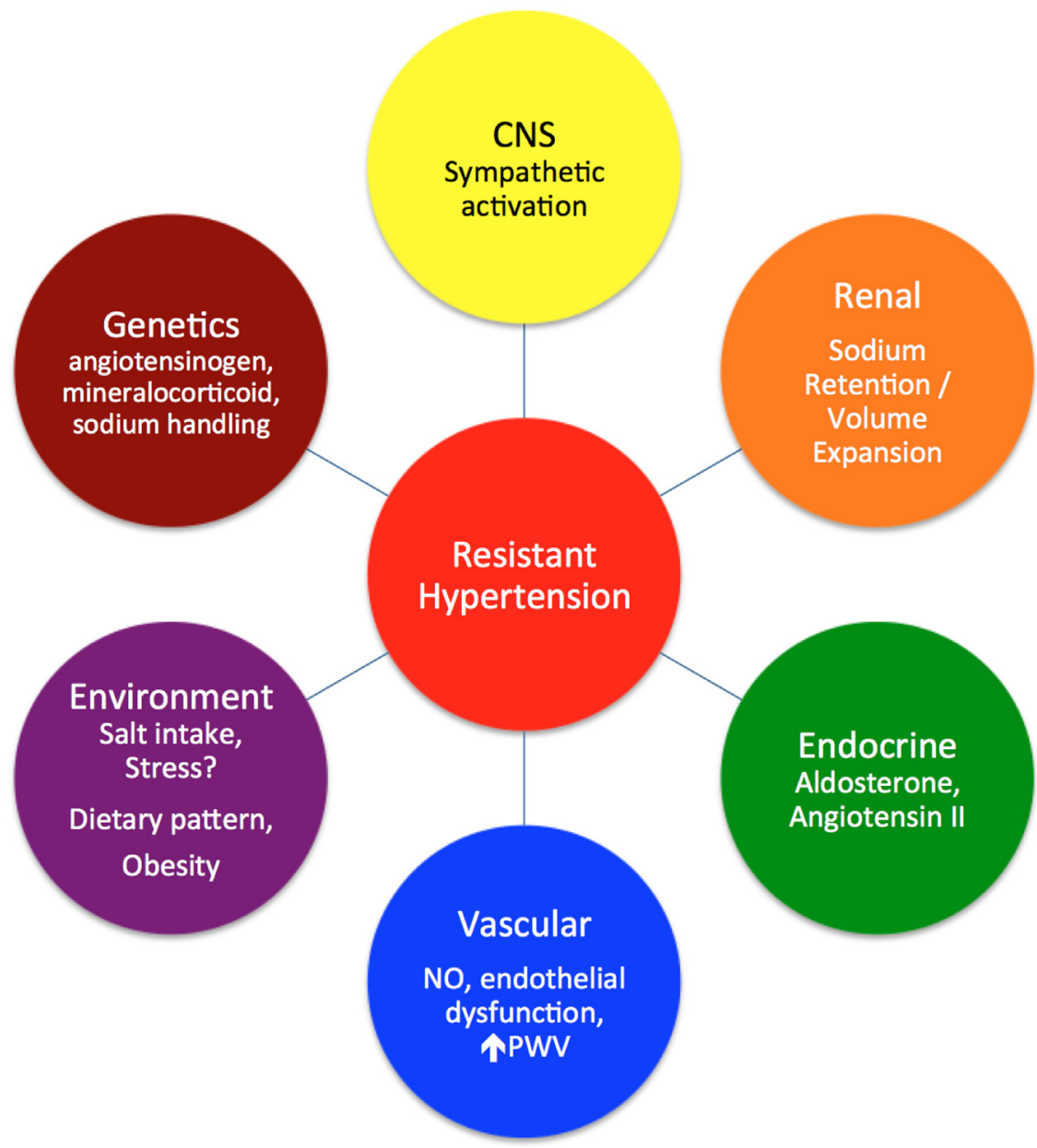

Figure 1. Pathophysiologic mechanisms of hypertension. Abbreviations: NO, nitric oxide; PWV, pulse wave velocity. 


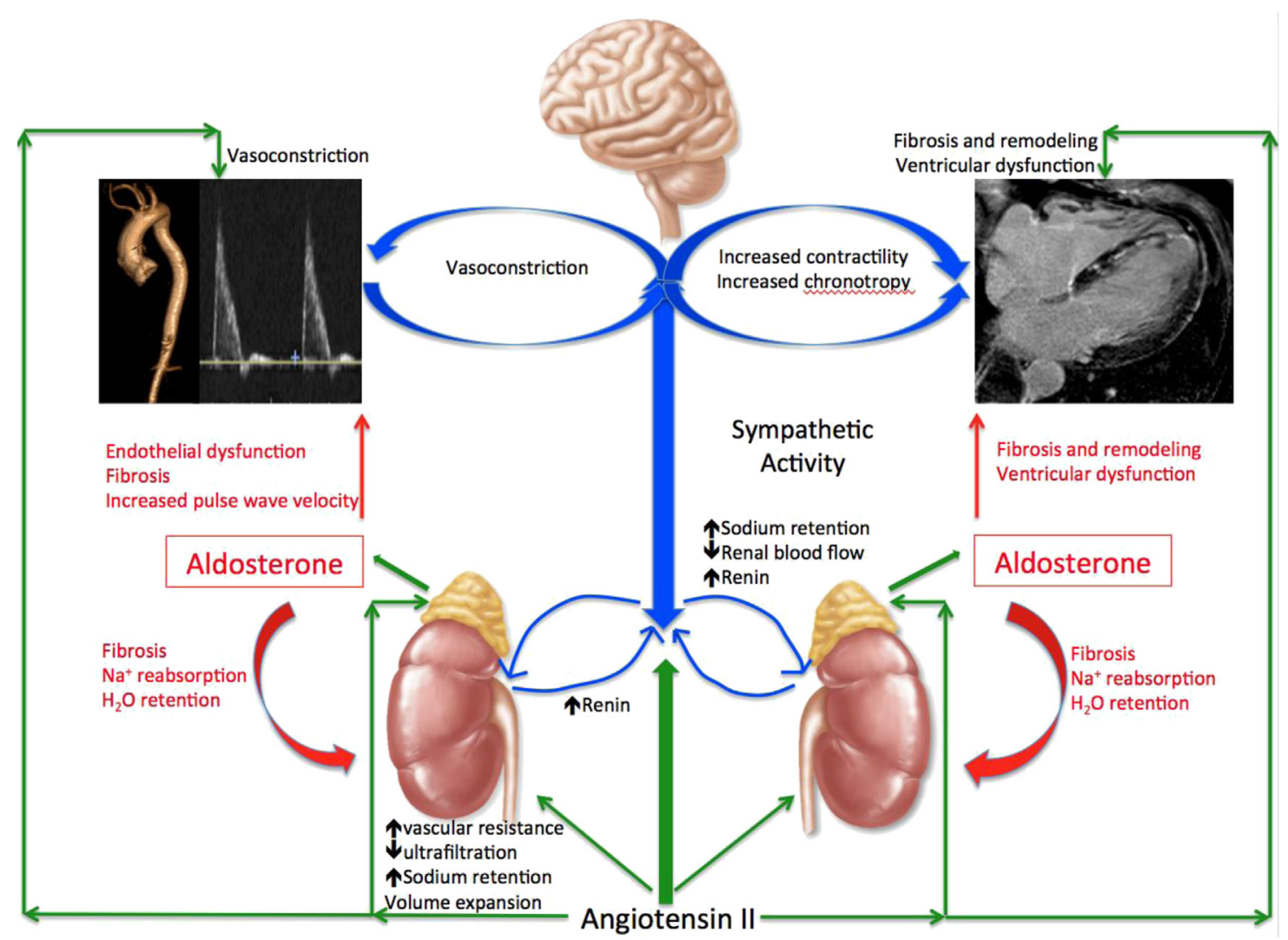

Figure 2. The renin-angiotensin-aldosterone system and sympathetic nervous systems in apparent treatment-resistant hypertension.

altered. In an animal model of renal injury, there is a 10fold increase in plasma aldosterone concentration that is dissociated from renin and angiotensin II levels. ${ }^{25}$ In a study comparing extracellular volume and plasma aldosterone concentration between 18 healthy controls and 18 patients with ESRD, patients with ESRD had higher plasma aldosterone concentrations at each level of extracellular fluid volume. ${ }^{26}$ Thus, CKD and its associated volume expansion are associated with elevated levels of plasma aldosterone that can exacerbate hypertension.

In addition to volume expansion, potential mechanisms of elevated aldosterone signaling in patients with CKD include hyperkalemia, NO synthase inhibition, and metabolic syndrome. Hyperkalemia directly stimulates aldosterone secretion. Independent of aldosterone, patients with $\mathrm{CKD}$ are known to have inhibitors of $\mathrm{NO}$ in their serum $^{27}$ and NO synthase inhibition results in increased aldosterone secretion. ${ }^{28}$ Finally, patients with metabolic syndrome have demonstrated adipose-mediated aldosterone excess. ${ }^{29}$ Taken together, these data suggest that CKD increases aldosterone levels and aldosterone in turn promotes hypertension through pleiotropic mechanisms.

\section{Arterial Stiffness, Volume Expansion, Aldosterone, and CKD}

Recently, multiple small studies have evaluated the role of arterial stiffness in aTRH and resistant hypertension. ${ }^{30,31}$ In a study of 90 consecutive patients with true resistant hypertension, patients with uncontrolled BP had higher aldosterone levels, greater left ventricular hypertrophy, and greater arterial stiffness. ${ }^{31}$ Additionally, vascular stiffness as assessed by aortic pulse-wave velocity is a known predictor of incident hypertension and increasing systolic blood pressure (SBP). ${ }^{32}$ Furthermore, increased aortic stiffness as assessed by pulse wave velocity is independently predictive of increase morbidity, CV mortality, and all-cause mortality in patients with hypertension. . $^{33,34}$ Although the exact mechanisms of aortic stiffening are incompletely understood, blood aldosterone synthase, ACE polymorphisms, ${ }^{35-37}$ and volume status have been independently associated with pulse wave velocity. ${ }^{38}$ Given the roles of aldosterone, angiotensin, and volume expansion in CKD, it is unsurprising that patients with CKD have increased aortic stiffness. ${ }^{39}$ In fact, in those with mild-to-severe CKD, aortic stiffness is associated with reductions in creatinine clearance independent of mean arterial pressure and other CV risk factors. ${ }^{40}$ In its role as a potential nexus between $\mathrm{CV}$ and renal pathologies underlying aTRH, arterial stiffness represents an alternative target of antihypertensive therapy. Currently available antihypertensives classes have differential effects on aortic stiffness as measured by pulse wave velocity, with beta-blockers and diuretics being less effective than angiotensin-converting enzyme inhibitor (ACE-I), calcium channel blockers, direct renin inhibitors, and aldosterone antagonists. ${ }^{41}$ Although further work is necessary to fully elucidate the contribution of arterial stiffness to treatment 
resistance, novel therapies directed at reversing arterial stiffness may hold promise in the treatment of resistant hypertension. ${ }^{42}$

\section{Role of the SNS in aTRH}

Recent animal and human studies have advanced our understanding of the role of the SNS in the pathogenesis of hypertension. The central SNS enervates the kidneys via efferent fibers originating in the thoracic and lumbar sympathetic trunks and receives signaling via afferent fibers from mechanoreceptors and chemoreceptors of the renal pelvis. Efferent fibers located in the adventitia of the renal vasculature release norepinephrine, adenosine triphosphate, and neuropeptide $Y$, causing increasing tubular sodium reabsorption, reduced renal blood flow, and increased renin release via $\alpha 1$ and $\beta 1$ adrenoceptor stimulation. ${ }^{43,44}$ Increased renin release subsequently causes further activation of the SNS and aldosterone production via the actions of angiotensin II (Fig 2), which increases BP.

\section{SNS and CKD}

Ishii and colleagues first demonstrated elevated plasma catecholamines in patients with CKD in $1983 .{ }^{45}$ After this observation, Converse and colleagues ${ }^{46}$ demonstrated the importance of afferent renal signaling by noting that bilateral nephrectomy reduces sympathetic activity to normal levels. Further studies have demonstrated that elevated sympathetic activity, mediated by renal damage and signaling via afferent renal nerves, is pathophysiologically important in patients with CKD. ${ }^{47}$ Lastly, Grassi and colleagues ${ }^{48}$ have shown that the level of adrenergic activation varies directly with the severity of CKD and, thus, inversely with the eGFR. The mechanisms by which renal injury causes activation of the SNS are incompletely understood; however, renal ischemia has been shown to cause mechanoreceptor and chemoreceptor release of adenosine, which in turn activate renal afferent neurons. In concert with the induction of the renin-angiotensin-aldosterone system (RAAS) and the production of angiotensin II, renal ischemia results in sympathetic stimulation. ${ }^{49}$

Patients with CKD are also known to have reduced NO bioavailability as a result of inflammation, oxidative stress, and uremic toxins. ${ }^{50}$ Because NO is responsible for tonic inhibition of the SNS, NO deficiency is thought to cause sympathetic overactivity and increased asymmetric dimethylarginine in patients with CKD, thus potentially contributing to hypertension. Notably, asymmetric dimethylarginine levels correlate with plasma norepinephrine levels, left ventricular hypertrophy, and left ventricular dysfunction. ${ }^{50}$ Thus, over time, multiple mechanisms of chronic SNS stimulation play a role in the development of hypertension.

\section{aTRH and CV Risk}

Although hypertension is estimated to be responsible for 17 million deaths per year worldwide ${ }^{1}$ and there is a doubling of $\mathrm{CV}$ risk with every $20 \mathrm{~mm} \mathrm{Hg}$ increase in $\mathrm{SBP}^{2} \mathrm{CV}$ outcomes in aTRH have only recently been inves- tigated. Data from patients with aTRH and vascular disease or risk factors for vascular disease within the REACH Registry indicate an elevated hazard ratio for the combined end point of $\mathrm{CV}$ death, myocardial infarction, and stroke $(1.32,95 \%$ confidence interval [CI] 1.05$1.67, P=.018)$ but not all cause mortality $(1.12,95 \% \mathrm{CI}$ $0.87-1.44, P=.38)$, $C V$ death $(1.38,95 \%$ CI $0.9-1.91$, $P=.06)$, fatal stroke $(1.42,95 \%$ CI $0.67-3.00, P=.36)$, nonfatal stroke $(1.40,95 \%$ CI $0.95-2.08, P=.089)$, or nonfatal MI $(0.87,95 \%$ CI $0.52-1.46, P=.60) .{ }^{11}$

In the Kaiser study of a broader population of aTRH with a median follow-up of 3.8 years, a composite of CV events, defined as death, myocardial infarction, congestive heart failure, stroke, or CKD, was significantly higher in the aTRH group after adjustment for patient and clinical characteristics (hazard ratio 1.47, 95\% CI 1.33-1.62). ${ }^{9}$ Notably, when the development of CKD was excluded, aTRH was no longer associated with an increased risk for adverse CV outcomes (adjusted hazard ratio 1.18; $95 \%$ CI 0.98 1.43, $P=.09$ ). ${ }^{9}$ Given the known link between BP and adverse CV outcomes, the Kaiser data suggest that either larger or higher risk cohorts of aTRH patients may be necessary to fully uncover the relationship between aTRH and CV outcomes.

Indeed, in a higher CV risk population of 436 patients with CKD, recent observational data by De Nicola and colleagues ${ }^{5}$ suggests that patients with resistant hypertension, defined as 24-hour mean ambulatory blood pressure monitoring (ABPM) of $125 / 75 \mathrm{~mm} \mathrm{Hg}$ or more and office BP of $130 / 80 \mathrm{~mm} \mathrm{Hg}$ or more, despite adherence to 3 or more full-dose antihypertensive drugs including a diuretic agent, or 4 or more drugs, experience higher rates of $C V$ events at 7 years of follow-up. CV events, defined as a composite of $\mathrm{CV}$ death or nonfatal $\mathrm{CV}$ event that required hospital stay (myocardial infarction, congestive heart failure, stroke, revascularization, peripheral vascular disease, and nontraumatic amputation) occurred in 25 $(21.2 \%), 8(25.8 \%)$, and $36(36.0 \%)$ patients in controlled, pseudoresistant, and true resistance groups, respectively $(P=.0007){ }^{5}$

\section{aTRH and Renal Risk}

Although hypertension is a known risk factor for CKD and ESRD, robust data investigating the relationship between aTRH and renal outcomes are scant. Among 436 patients with CKD and 7 years of follow-up, De Nicola and colleagues ${ }^{5}$ noted a stepwise progression in the risk of renal events, including ESRD or death, among patients with controlled (21.2\%), pseudoresistant $(16.1 \%)$, and true resistant hypertension $(57 \%$; $P<.0001)$. A larger analysis from the REGARDS study with 6.4 years of median follow-up found that the hazard ratio for ESRD comparing hypertensive participants with aTRH with those without aTRH was 6.32 (95\% CI 4.30-9.30). ${ }^{51}$ Furthermore, in a separate clinic-based study of 300 patients with CKD, aTRH was associated with an increased risk of a composite outcome of dialysis, kidney transplantation, or death (hazard ratio $=1.85,95 \%$ CI 1.13-3.03). ${ }^{52}$ Thus, the current literature suggests a strong association between aTRH and renal outcomes and a weaker 
association between aTRH and stroke or cardiac outcomes compared with non-aTRH.

\section{Treatment of aTRH}

In light of the risk of adverse $\mathrm{CV}$ outcomes associated with aTRH, a comprehensive treatment plan must address the multifactorial aspects of disease pathogenesis.

\section{Salt Restriction}

Although salt restriction has been shown to reduce BP in patients with and without hypertension, ${ }^{53}$ the role of salt restriction in the treatment of aTRH has been less well investigated. An analysis from the REGARDS cohort indicated that among patients with aTRH, the prevalence of low-Dietary Approaches to Stop Hypertension (DASH) diet score was $23.1 \%$, and $25.4 \%$ had high $\mathrm{Na} / \mathrm{K}$ intake. After adjustment for age, sex, race, and geographic region of residence, neither of these lifestyle factors were associated with aTRH. Nevertheless, a single, small, randomized crossover study of 12 patients with aTRH compared a low sodium diet ( $50 \mathrm{mmol} / \mathrm{d} \times 7$ days) (vs) a high sodium diet $(250 \mathrm{mmol} / \mathrm{d} \times 7$ days $) .{ }^{54}$ Low compared with highsalt diet decreased office SBP and diastolic blood pressure (DBP) by 22.7 and $9.1 \mathrm{~mm} \mathrm{Hg}$, respectively. Plasma renin activity increased, whereas brain natriuretic peptide and creatinine clearance decreased during low-salt intake, indicative of intravascular volume reduction. Although no data for salt restriction in patients with CKD and aTRH exist, given that patients with CKD have impaired sodium excretion and subsequent volume expansion, it is likely that salt restriction will be effective in reducing BP among patients with CKD and aTRH.

Although other nonpharmacologic interventions for BP lowering, such as weight loss in overweight/obesity and the DASH dietary pattern, ${ }^{55}$ have not been specifically tested in aTRH, given that their effects are greatest in those with the highest $\mathrm{BP}$, one would expect these lifestyle treatments to be effective in aTRH as well. Similarly, lifestyle treatments have not been tested in patients with CKD. In light of the tendency for malnutrition and hyperkalemia in CKD, which could be exacerbated by weight loss and DASH dietary pattern, respectively, additional research is needed before recommending these interventions for patients with aTRH in the presence of CKD.

\section{Pharmacotherapy: Diuretics}

Given consistent evidence of reduced morbidity and mortality with diuretic treatment of hypertension, all major societal guidelines currently emphasize the importance of diuretic therapy. ${ }^{6,7,56,57}$ The apparent role of volume expansion in the pathophysiology of aTRH suggests that diuretics may be particularly important in this setting. Choice of diuretic class is primarily determined by eGFR, with thiazide diuretics being used preferentially for patients with eGFR of $30 \mathrm{~mL} / \mathrm{min} / 1.73 \mathrm{~m}^{2}$ or more and loop diuretics preferred for patients with lesser eGFR. ${ }^{58}$ Among thiazide diuretics, significant controversy exists regarding the comparative effectiveness of indapamide, hydrochlorothiazide, and chlorthalidone. Currently, no randomized head-to-head comparison of these agents exists, and various meta-analyses have found either no difference between agents ${ }^{59,60}$ or a benefit in ambulatory $\mathrm{BP}$ reduction and $\mathrm{CV}$ outcomes with chlorthalidone. ${ }^{61,62}$ As a result, although the AHA Scientific Statement on Resistant Hypertension preferentially recommends chlorthalidone ${ }^{6}$ and the American Society of Hypertension position article notes that chlorthalidone is more potent and longer acting than hydrochlorothiazide, ${ }^{7}$ the European Society of Hypertension suggest there is insufficient data to recommend 1 agent over the other. ${ }^{57}$ However, because of its superior potency, longer half-life, and evidence base for improved CV outcomes, we advocate the use of chlorthalidone as the diuretic agent of choice in patients with eGFR of $30 \mathrm{~mL} / \mathrm{min} / 1.73 \mathrm{~m}^{2}$ or more and the use of loop diuretics in patients with eGFR less than $30 \mathrm{~mL} / \mathrm{min} / 1.73 \mathrm{~m}^{2}$.

Though the comparative effectiveness of thiazide diuretics for the treatment of hypertension and aTRH remains an open question, diuretic use and dosing appears to be a stumbling block to achieve BP control in aTRH. Despite the recommended use of diuretics, a study of patients referred to the Rush University Hypertension Clinic noted that lack of BP control was most often because of suboptimal diuretic use, suboptimal diuretic dosing, or inappropriate diuretic class for eGFR. ${ }^{63}$ Subsequent observational analyses of NHANES data (2005 to 2008) indicate that only $83.9 \%(76.0 \%-89.5 \%)$ of patients with uncontrolled $\mathrm{BP}$ on 3 or more medications were taking a diuretic. $^{10}$

\section{Pharmacotherapy: Nondiuretics}

Beyond the role of diuretics, little data exist to guide practitioners regarding the choice of subsequent antihypertensives in patients with aTRH. Currently, there are no clinical trials assessing the comparative effectiveness of antihypertensive classes in patients with aTRH and CKD. However, a meta-analysis of randomized controlled trials of $\mathrm{BP}$ lowering and major CV events in patients with CKD and hypertension found that there was no significant difference between agents in reduction of $\mathrm{CV}$ events after taking into account small differences in BP reductions achieved. ${ }^{64}$

Given the observed prevalence of hyperaldosteronism among patients with aTRH, existing data regarding addon antihypertensive therapy has focused on the role of aldosterone antagonism. Nonrandomized trial data for this strategy include a study of 76 patients both with and without hyperaldosteronism referred to a hypertension clinic while on a regimen of a diuretic plus an ACE-I or angiotensin receptor blocker. Addition of low-dose spironolactone was associated with a 21/10 $\mathrm{mm} \mathrm{Hg}$ reduction in BP at 6 weeks and 25/12 $\mathrm{mm} \mathrm{Hg}$ reduction at 6-month follow-up. ${ }^{65}$ Furthermore, in a secondary analysis of Anglo-Scandinavian Cardiac Outcomes Trial Blood Pressure Lowering Arm, spironolactone was associated with a $21.9 / 9.5 \mathrm{~mm} \mathrm{Hg}$ reduction in BP (95\% CI 20.8 to $23.0 /$ 9.0 to $10.1 \mathrm{~mm} \mathrm{Hg} ; P<.001) .{ }^{66}$ The subsequent Addition of Spironolactone in Patients With Resistant Arterial Hypertension trial randomized patients with aTRH to spironolactone $25 \mathrm{mg}$ (vs) placebo and demonstrated a mean decrease in daytime systolic ambulatory BP of $5.4 \mathrm{~mm}$ $\mathrm{Hg}(P=.02)$ and 24-hour systolic ambulatory BP $(9.8 \mathrm{~mm} \mathrm{Hg}, P=.01)$ and night-time systolic ambulatory $\mathrm{BP}(8.6 \mathrm{~mm} \mathrm{Hg}, P=.01)$ at 8 weeks of therapy. 
In light of the contribution of hyperaldosteronism and upregulation of the renin-aldosterone system to the pathophysiology of aTRH, Bobrie and colleagues conducted a prospective, randomized trial of sequential diuretic use (vs) sequential RAAS blockade. Patients on maximum tolerated doses of thiazide diuretic, angiotensin receptor blocker, and calcium channel blocker and mean daytime ambulatory SBP of $135 \mathrm{~mm} \mathrm{Hg}$ or more were randomized to either sequential nephron blockade with stepped addition of aldosterone antagonist, loop diuretic, and potassium sparing diuretic or sequential RAAS blockade with ACE-I and beta-blocker. Notably, patients with contraindications to the study medication or eGFR less than $40 \mathrm{~mL} /$ $\mathrm{min} / 1.73 \mathrm{~m}^{2}$ were excluded. After 12 weeks of therapy, mean daytime ambulatory SBP fell by $18 \mathrm{~mm} \mathrm{Hg}$ $(150 \pm 14$ to $132 \pm 17 \mathrm{~mm} \mathrm{Hg})$ with sequential nephron blockade and by $8 \mathrm{~mm} \mathrm{Hg}(151 \pm 13$ to $143 \pm 17 \mathrm{~mm}$ $\mathrm{Hg}$ ) with sequential RAAS blockade $(P<.0001)$. Serious adverse events did not differ significantly between groups. ${ }^{67}$ Thus, sequential diuretic use appeared to be more effective than sequential RAAS blockade for BP lowering without any excess in adverse events.

The safety and efficacy of the use of aldosterone antagonists in patients with stages 2 and 3 CKD has been evaluated in 2 small retrospective studies. ${ }^{68,69}$ In 32 patients with Stage 3 CKD referred to a hypertension clinic and treated with spironolactone $(n=32)$ or eplerenone $(n=4)$, the mean SBP dropped from $162 \pm 22$ to $138 \pm 14 \mathrm{~mm} \mathrm{Hg}(P<.0001)$ and the mean DBP dropped from $87 \pm 17$ to $74 \pm 12 \mathrm{~mm} \mathrm{Hg}$ over a median followup of 312 days. Notably, serum potassium increased from $4.0 \pm 0.5$ to $4.4 \pm 0.5 \mathrm{mEq} / \mathrm{L}(P=.0001)$, with the highest value being $5.8 \mathrm{mEq} / \mathrm{L}$. There was 1 case of acute renal failure and 3 cases of significant hyperkalemia. ${ }^{68}$ Additionally, a prespecified secondary analysis of patients with heart failure with reduced ejection fraction and eGFR range of 30 to $59 \mathrm{~mL} / \mathrm{min} / 1.73 \mathrm{~m}^{2}$ treated with eplerenone showed an increased risk of potassium greater than $5.5 \mathrm{mmol} / \mathrm{L}$ but not of potassium greater than $6.0 \mathrm{mmol} / \mathrm{L}$ or hospitalization for hyperkalemia or discontinuation of eplerenone. ${ }^{70}$ Consequently, in patients with eGFR of $30 \mathrm{~mL} / \mathrm{min} / 1.73 \mathrm{~m}^{2}$ or more and not limited by baseline hyperkalemia, we recommend the addition of an aldosterone antagonist (spironolactone or eplerenone) as an add-on agent with ongoing potassium and creatinine monitoring.

\section{Interventional Therapy: Device Based Sympathetic Modulation \\ Renal Denervation}

Existing classes of pharmacotherapy address the pathologic contributions of volume expansion, upregulated RAAS, and hyperaldosteronism that lead to renal microvascular injury and aTRH. Until recently, beta-blockers, central alpha agonists, and peripheral alpha antagonists were the only clinically available antihypertensives thought to block sympathetic overactivity. However, studies of the effects of beta-blockade on markers of SNS activity have shown varying levels of effectiveness of SNS blockade. ${ }^{71,72}$ Additionally, administration of the central alpha-adrenergic agonist clonidine has been demonstrated to reduce plasma catecholamines in animals and humans. ${ }^{73,74}$ However, remaining sympathetic activity, despite the presence of clonidine, may continue to contribute to hypertension. ${ }^{75}$

Before modern pharmacotherapy, surgical radical sympathectomy was used to treat malignant hypertension. In an observational study, 1266 patients who underwent nonselective open surgical sympathectomy had improved survival and BP control compared with 467 patients treated conventionally. ${ }^{76}$ However, because of high surgical morbidity and mortality and the advent of effective pharmacotherapy for hypertension, surgical sympathectomy was largely abandoned.

Contemporary minimally invasive vascular intervention techniques have allowed for delivery of radiofrequency or ultrasound energy via steerable catheters to produce selective renal sympathetic denervation. Pathology studies in swine 6 months after renal denervation indicate that first-generation devices currently available in Europe produce $10 \%-25 \%$ fibrosis of the intimal media and underlying adventitia. This results in renal nerve fibrosis and thickening of the epineurium and perineurium with minimal disruption of the external elastic lamina and no significant smooth muscle hyperplasia or inflammation. ${ }^{77}$ Further studies of renal denervation in animal models of hypertension have demonstrated marked reductions in $\mathrm{BP}^{78,79}$

Although multiple device companies have been approved in Europe based on nonrandomized observational studies, randomized human data examining renal denervation in aTRH have until recently been limited to the SYMPLICITY HTN-2 trial. In this international study, 106 patients with eGFR of $45 \mathrm{~mL} / \mathrm{min} / 1.73 \mathrm{~m}^{2}$ or more and aTRH, defined as a stable drug regimen of $3+$ antihypertensive medications and an office SBP of $160 \mathrm{~mm} \mathrm{Hg}$, were randomized 1:1 to continued medical therapy (vs) catheter-based renal denervation. Baseline $\mathrm{BP}$ was $178 / 96 \mathrm{~mm} \mathrm{Hg}$ and $178 / 97 \mathrm{~mm} \mathrm{Hg}$ in the denervation and control group, respectively. At 6 months, office BP decreased by $32 / 12 \mathrm{~mm} \mathrm{Hg}( \pm 23 / 11 \mathrm{~mm} \mathrm{Hg})$ in the renal denervation group $(P<.0001)$ and by $1 / 0 \mathrm{~mm} \mathrm{Hg}( \pm 21 /$ $10 \mathrm{~mm} \mathrm{Hg})$ in the medical therapy group $(P=.77$ systolic and $P=.83$ diastolic). Intergroup differences in $\mathrm{BP}$ were 33 of $11(P<.0001)$ and $84 \%$ of patients who underwent renal denervation had a reduction in SBP of $10 \mathrm{mmHg}$ or more compared with $35 \%$ of controls $(P<.0001) .{ }^{80}$ Importantly, adverse events included 1 case of femoral artery pseudoaneurysm, 7 cases of intraprocedural bradycardia necessitating atropine, and 1 case of postprocedural hypotension requiring a reduction in antihypertensive doses. The effect appeared to be durable at 12 months, and patients who crossed over from the medical therapy arm to the denervation arm at 6 months experienced a similar BP response to the original denervation arm. ${ }^{81}$

Although SYMPLICITY HTN-2 excluded patients with eGFR less than $45 \mathrm{~mL} / \mathrm{min} / 1.73 \mathrm{~m}^{2}$, nonrandomized, observational data suggest that renal denervation is effective and safe in patients with lower eGFR. ${ }^{82,83}$ Bilateral renal denervation in 15 patients with Stage 3 to 4 CKD (mean eGFR $31 \mathrm{~mL} / \mathrm{min} / 1.73 \mathrm{~m}^{2}$ ) and aTRH resulted in mean changes in office SBP and DBP at 1, 3, 6, and 
12 months of $-34 /-14,-25 /-11,-32 /-15$, and $-33 /$ $-19 \mathrm{~mm} \mathrm{Hg}$, respectively. $\mathrm{CO}_{2}$ angiography was used in 6 patients to limit contrast exposure, but no significant change in eGFR was noted with renal denervation, irrespective of the use of $\mathrm{CO}_{2}$ angiography. ${ }^{82}$

Importantly, despite the apparent efficacy of renal denervation for the treatment of aTRH in the SYMPLICITY HTN-2 trial and in small, proof-of concept studies in CKD, several important study design limitations affect interpretation of the results of these studies. Although randomized, patients in the medical therapy (control arm) of SYMPLICITY HTN-2 did not undergo sham procedures and, thus, placebo effect and measurement bias cannot be ruled out. Second, patients with secondary hypertension and white coat hypertension were not explicitly excluded, and third, 24-hour ambulatory SBP was reduced by only $11 \mathrm{~mm} \mathrm{Hg}$. Although the discrepancy in office and ambulatory BP reduction may be the result of a failure to exclude white coat hypertension, similar discrepancies in antihypertensive drug trials have been because of a lack of blinding and lack of placebo control. ${ }^{84}$ As a result, institution of blinding and placebo control in trial design has attenuated office BP reductions and has left ambulatory $\mathrm{BP}$ reductions unchanged in antihypertensive drug trials.

In response to these concerns, the SYMPLICITY HTN-3 trial randomized 535 patients with eGFR of $45 \mathrm{~mL} / \mathrm{min} /$ $1.73 \mathrm{~m}^{2}$ or more and aTRH, as defined by treatment with 3 optimally dosed antihypertensives (including a diuretic), SBP of 160 or more, and average ABPM of $135 \mathrm{~mm} \mathrm{Hg}$ or more, to catheter-based renal denervation or sham procedure. ${ }^{85}$ The prespecified primary efficacy end point was the difference in change in office $\mathrm{BP}$ from baseline to 6 months between the denervation and sham procedure arms. The secondary efficacy end point was change in mean 24-hour ambulatory BP from baseline to 6 months between the denervation and sham procedure arms. The primary safety end point was the incidence of major adverse events. There was no significant difference between groups in the change in office $(-2.39 \mathrm{~mm} \mathrm{Hg} ; 95 \%$ $\mathrm{CI}-6.89$ to $2.12 ; P=.26)$ or ambulatory BP $(-1.96 \mathrm{~mm}$ $\mathrm{Hg} ; 95 \% \mathrm{CI}-4.97$ to $1.06 ; P=.98)$ at 6 months. Additionally, there was no significant difference in major adverse events (death, end-stage renal disease, embolic event causing end organ damage, vascular complications, hypertensive crisis within 30 days, or new renal artery stenosis of $>70 \%$ within 6 months) between groups ( $1.4 \%$ (vs) $0.6 \%$, difference of $0.8 \% ; 95 \% \mathrm{CI}-0.9 \%$ to $2.5 \%$; $P=.67)$.

Although superior study design may explain the discrepancy between the results of SYMPLICITY HTN-3 and previous studies of renal denervation, a number of other factors may also contribute. First, analysis of the SYMPLICITY HTN-3 results reveals that office BPs changed by $-14.13 \pm 23.93 \mathrm{~mm} \mathrm{Hg}$ in the denervation arm and $-11.74 \pm 25.94 \mathrm{~mm} \mathrm{Hg}$ in the sham arm, indicating both a significant placebo effect and significant BP variability. Six-month changes in mean 24-hour ambulatory BP indicated similar variability and placebo effect $(-6.75 \pm 15.11 \mathrm{~mm} \mathrm{Hg}$ (vs) $-4.79 \pm 17.25 \mathrm{~mm} \mathrm{Hg})$. Variability in BP change may indicate that renal denervation is effective for some individuals, and indeed, subgroup analysis of nonblack individuals revealed a statistically signif- icant $-6.63 \mathrm{~mm} \mathrm{Hg}(-11.81$ to $-1.44 \mathrm{~mm} \mathrm{Hg} ; P=.01)$ drop in office BP. ${ }^{86}$

Second, there is currently no clinically available method for determining the efficacy of energy delivery in causing renal denervation. Although early animal and human studies have used norepinephrine spillover and muscle sympathetic nerve activity to document reductions in sympathetic tone after denervation, later studies, such as SYMPLICITY HTN-2 and SYMPLICITY HTN-3, have not used these tests because of their limited availability. As a result, it is unclear whether effective denervation was achieved in the SYMPLICITY HTN-3 trial, although the same catheter system was used as in previous studies. Interestingly, renal artery notching, a marker of sufficient energy delivery to cause arterial spasm, was only noted in $58.6 \%$ of patients. ${ }^{86}$

Regardless of the reason for the discrepancy between SYMPLICITY HTN-3 and earlier studies, these sobering results signify that clinical implementation of RDN will require 1 or both of the following: (1) significant improvement in achieving clinical denervation and (2) identification of subgroups of patients for whom this technique is effective. Additionally, clinicians, patients, and society as a whole will need to balance the magnitude of any BP effect against the risks and costs of the procedure. As a result, future avenues of research should focus on techniques to provide operator feedback regarding the efficacy of denervation and/or testing RDN in subgroup populations.

\section{Carotid Baroreceptor Activation}

Sympathetic modulation via carotid baroreceptor activation has also been investigated in patients with aTRH. Current-generation carotid baroreceptor devices comprising electrodes implanted on the carotid body connected to a generator implanted subcutaneously. The Rheos Pivotal Trial implanted 265 patients with aTRH and subsequently randomized them 2:1 to baroreceptor activation therapy for 6 months (group A) or delayed baroreceptor activity after 6 months (group B). The trial met a secondary end point of sustained efficacy at 6 months (42\% in group A (vs) $24 \%$ in group B achieved SBP of $140 \mathrm{~mm} \mathrm{Hg}$ or less at 6 months $(P=.005)$. However, procedural complications included nerve injury with residual deficit $(4.8 \%)$, respiratory complications $(2.6 \%)$, and wound complications $(2.6 \%) .{ }^{87} \mathrm{~A}$ nonrandomized follow-up analysis documented $76 \%$ clinical response at 22 to 55 months with an average BP reduction of 35/ $16 \mathrm{~mm} \mathrm{Hg}{ }^{88}$ Of note, serum creatinine increased from 0.86-0.92 $\mathrm{mg} / \mathrm{dL}$, and eGFR decreased from $92-87 \mathrm{~mL} /$ $\mathrm{min} / 1.73 \mathrm{~m}^{2}$ in group A at 6 months $(P<.05)$. Although the drop in glomerular filtration was most notable in patients with the greatest reduction in $\mathrm{BP}$, the average eGFR drops were not clinically significant. ACR was unchanged and after 12 months of therapy, there was no further reduction in eGFR in this group. ${ }^{89}$ Although the authors hypothesized that the observed decrease in eGFR was related to decreased renal perfusion pressure because of improved BP control, further data will be necessary to establish the safety of carotid baroreceptor activation in CKD. 


\section{Conclusions}

Resistant hypertension is a common and growing problem. Absent the exclusion of pseudoresistance by assessment of home or ambulatory BPs and medication compliance, it is known as aTRH. Although not all patients with aTRH have CKD and not all patients with CKD have aTRH, CKD and albuminura are significantly associated with aTRH. ATRH appears to confer an increased risk of adverse renal outcomes and, to a lesser extent, adverse $\mathrm{CV}$ outcomes. Although event rates appear to be highest in patients with aTRH and known vascular disease or risk factors for vascular disease, patients with aTRH and CKD may have higher event rates than those with aTRH alone.

Treatment of the patient with aTRH is challenging, in part because of a paucity of clinical trial data to guide selection of combination therapies. Although not specifically tested in patients with aTRH, a sodium-restricted diet along with weight loss and moderation of alcohol intake still form the mainstay of nonpharmacologic therapies. Patients with CKD and aTRH present unique treatment hurdles with respect to diuretic selection and eligibility for addon therapy with aldosterone antagonists.

In the absence of trial data specific to patients with aTRH, the choice of individual antihypertensive agents in patients with aTRH is based on a combination of addressing each of the major pathophysiologic contributors to aTRH and using therapies with proven benefit on $\mathrm{CV}$ outcomes in patients with non-aTRH. In the presence of coexisting CKD, treatment is also driven by CKD pathophysiology and safety concerns. Device-based therapies, including renal denervation and carotid baroreceptor activation, warrant further investigation.

\section{References}

1. Lim SS, Vos T, Flaxman AD, et al. A comparative risk assessment of burden of disease and injury attributable to 67 risk factors and risk factor clusters in 21 regions, 1990-2010: a systematic analysis for the Global Burden of Disease Study 2010. Lancet. 2012;15:2224-2260.

2. Yoon PWG, Cathleen D, George MG, Wall HK. Control of hypertension among adults - National Health and Nutrition Examination Survey, United States, 2005-2005. Morb Mortal Wkly Rep. 2012;61(2): $19-25$.

3. Lawes CM, Vander Hoorn S, Rodgers A. Global burden of bloodpressure-related disease, 2001. Lancet. 2008;371(9623):1513-1518.

4. Tsioufis C, Kasiakogias A, Kordalis A, et al. Dynamic resistant hypertension patterns as predictors of cardiovascular morbidity: a 4year prospective study. J Hypertens. 2014;32(2):415-422.

5. De Nicola L, Gabbai FB, Agarwal R, et al. Prevalence and prognostic role of resistant hypertension in chronic kidney disease patients. $J$ Am Coll Cardiol. 2013;61(24):2461-2467.

6. Calhoun DA, Jones D, Textor S, et al. Resistant hypertension: diagnosis, evaluation, and treatment: a scientific statement from the American Heart Association Professional Education Committee of the Council for High Blood Pressure Research. Circulation. 2008; 117(25):e510-e526.

7. Weber MA, Schiffrin EL, White WB, et al. Clinical practice guidelines for the management of hypertension in the community: a statement by the American Society of Hypertension and the International Society of Hypertension. J Clin Hypertens. 2014;16(1):14-26.

8. Vemulapalli S, Ard J, Bakris GL, et al. Proceedings from Duke resistant hypertension think tank. Am Heart J. 2014;167:775-788.e1.
9. Daugherty SL, Powers JD, Magid DJ, et al. Incidence and prognosis of resistant hypertension in hypertensive patients. Circulation. 2012;125(13):1635-1642.

10. Egan BM, Zhao Y, Axon RN, Brzezinski WA, Ferdinand KC. Uncontrolled and apparent treatment resistant hypertension in the United States, 1988 to 2008. Circulation. 2011;124(9):1046-1058.

11. Kumbhani DJ, Steg PG, Cannon CP, et al. Resistant hypertension: a frequent and ominous finding among hypertensive patients with atherothrombosis. Eur Heart J. 2013;34(16):1204-1214.

12. Crews DC, Plantinga LC, Miller ER III, et al. Prevalence of chronic kidney disease in persons with undiagnosed or prehypertension in the United States. Hypertension. 2010;55(5):1102-1109.

13. Tanner RM, Calhoun DA, Bell EK, et al. Prevalence of apparent treatment-resistant hypertension among individuals with CKD. Clin J Am Soc Nephrol. 2013;8(9):1583-1590.

14. Johnson RJ, Herrera-Acosta J, Schreiner GF, Rodríguez-Iturbe B. Subtle acquired renal injury as a mechanism of salt-sensitive hypertension. N Engl J Med. 2002;346(12):913-923.

15. Oparil S, Zaman MA, Calhoun DA. Pathogenesis of hypertension. Ann Intern Med. 2003;139(9):761-776.

16. Suga SI, Phillips MI, Ray PE, et al. Hypokalemia induces renal injury and alterations in vasoactive mediators that favor salt sensitivity. Am J Physiol Renal Physiol. 2001;281(4):F620-F629.

17. Ray PE, Suga S, Liu XH, Huang X, Johnson RJ. Chronic potassium depletion induces renal injury, salt sensitivity, and hypertension in young rats. Kidney Int. 2001;59(5):1850-1858.

18. Lynch AI, Irvin MR, Davis BR, Ford CE, Eckfeldt JH, Arnett DK. Genetic and adverse health outcome associations with treatment resistant hypertension in GenHAT. Int J Hypertens. 2013;2013: 578578.

19. Fontana V, de Faria AP, Barbaro NR, et al. Modulation of aldosterone levels by $-344 \mathrm{c} / \mathrm{t}$ cyp11b2 polymorphism and spironolactone use in resistant hypertension. J Am Soc Hypertens. 2014;8:146-151.

20. Calhoun DA, Nishizaka MK, Zaman MA, Thakkar RB, Weissmann P. Hyperaldosteronism among black and white subjects with resistant hypertension. Hypertension. 2002;40(6):892-896.

21. Briet M, Schiffrin EL. Vascular actions of aldosterone. J Vasc Res. 2013;50(2):89-99.

22. Shibata H, Itoh H. Mineralocorticoid receptor-associated hypertension and its organ damage: clinical relevance for resistant hypertension. Am J Hypertens. 2012;25(5):514-523.

23. Park S, Kim JB, Shim CY, et al. The influence of serum aldosterone and the aldosterone-renin ratio on pulse wave velocity in hypertensive patients. J Hypertens. 2007;25(6):1279-1283.

24. Vasan RS, Evans JC, Larson MG, et al. Serum aldosterone and the incidence of hypertension in nonhypertensive persons. $N$ Engl J Med. 2004;351(1):33-41.

25. Greene EL, Kren S, Hostetter TH. Role of aldosterone in the remnant kidney model in the rat. J Clin Invest. 1996;98(4):1063-1068.

26. Volk MJ, Bomback AS, Klemmer PJ. Mineralocorticoid receptor blockade in chronic kidney disease. Curr Hypertens Rep. 2011;13(4): 282-288.

27. Zoccali C, Bode-Boger S, Mallamaci F, et al. Plasma concentration of asymmetrical dimethylarginine and mortality in patients with end-stage renal disease: a prospective study. Lancet. 2001; 358(9299):2113-2117.

28. Rocha R, Stier CT Jr, Kifor I, et al. Aldosterone: a mediator of myocardial necrosis and renal arteriopathy. Endocrinology. 2000;141(10):3871-3878.

29. Goodfriend TL, Calhoun DA. Resistant hypertension, obesity, sleep apnea, and aldosterone: theory and therapy. Hypertension. 2004;43(3):518-524.

30. Pabuccu T, Baris N, Ozpelit E, Akdeniz B, Guneri S. The relationship between resistant hypertension and arterial stiffness. Clin Exp Hypertens. 2012;34(1):57-62.

31. Martins LC, Figueiredo VN, Quinaglia T, et al. Characteristics of resistant hypertension: ageing, body mass index, hyperaldosteronism, 
cardiac hypertrophy and vascular stiffness. I Hum Hypertens. 2011;25(9):532-538.

32. Najjar SS, Scuteri A, Shetty V, et al. Pulse wave velocity is an independent predictor of the longitudinal increase in systolic blood pressure and of incident hypertension in the Baltimore Longitudinal Study of Aging. J Am Coll Cardiol. 2008;51(14):1377-1383.

33. Laurent $S$, Boutouyrie $P, A s m a r ~ R$, et al. Aortic stiffness is an independent predictor of all-cause and cardiovascular mortality in hypertensive patients. Hypertension. 2001;37(5):1236-1241.

34. Laurent S, Katsahian S, Fassot C, et al. Aortic stiffness is an independent predictor of fatal stroke in essential hypertension. Stroke. 2003;34(5):1203-1206.

35. Blacher J, Kakou A, Lacombe JM, Safar ME. Preferential association of aldosterone synthase gene polymorphism with central blood pressure and wave reflections in hypertensive individuals. J Hum Hypertens. 2010;24(4):291-299.

36. Safar ME, Cattan V, Lacolley P, et al. Aldosterone synthase gene polymorphism, stroke volume and age-related changes in aortic pulse wave velocity in subjects with hypertension. J Hypertens. 2005;23(6):1159-1166.

37. Balkestein EJ, Staessen JA, Wang JG, et al. Carotid and femoral artery stiffness in relation to three candidate genes in a white population. Hypertension. 2001;38(5):1190-1197.

38. Di Iorio B, Nazzaro P, Cucciniello E, Bellizzi V. Influence of haemodialysis on variability of pulse wave velocity in chronic haemodialysis patients. Nephrol Dial Transplant. 2010;25(5):1579-1583.

39. Safar ME, London GM, Plante GE. Arterial stiffness and kidney function. Hypertension. 2004;43(2):163-168.

40. Doyle A, Mark PB, Johnston N, et al. Aortic stiffness and diastolic flow abnormalities in end-stage renal disease assessed by magnetic resonance imaging. Nephron Clin Pract. 2008;109(1):c1-c8.

41. Dudenbostel T, Glasser SP. Effects of antihypertensive drugs on arterial stiffness. Cardiol Rev. 2012;20(5):259-263.

42. Zieman SJ, Melenovsky V, Clattenburg L, et al. Advanced glycation endproduct crosslink breaker (alagebrium) improves endothelial function in patients with isolated systolic hypertension. J Hypertens. 2007;25(3):577-583.

43. DiBona GF. Physiology in perspective: the wisdom of the body. Neural control of the kidney. Am J Physiol Regul Integr Comp Physiol. 2005;289(3):R633-R641.

44. Vonend O, Okonek A, Stegbauer J, Habbel S, Quack I, Rump LC. Renovascular effects of sympathetic cotransmitters ATP and NPY are age-dependent in spontaneously hypertensive rats. Cardiovasc Res. 2005;66(2):345-352.

45. Ishii M, Ikeda T, Takagi M, et al. Elevated plasma catecholamines in hypertensives with primary glomerular diseases. Hypertension. 1983;5(4):545-551.

46. Converse RL Jr, Jacobsen TN, Toto RD, et al. Sympathetic overactivity in patients with chronic renal failure. $N$ Engl $J$ Med. 1992;327(27):1912-1918.

47. Hausberg M, Kosch M, Harmelink P, et al. Sympathetic nerve activity in end-stage renal disease. Circulation. 2002;106(15):1974-1979.

48. Grassi G, Quarti-Trevano F, Seravalle G, et al. Early sympathetic activation in the initial clinical stages of chronic renal failure. Hypertension. 2011;57(4):846-851.

49. Carlson SH, Wyss JM. Neurohormonal regulation of the sympathetic nervous system: new insights into central mechanisms of action. Curr Hypertens Rep. 2008;10(3):233-240.

50. Baylis C. Nitric oxide deficiency in chronic kidney disease. Am J Physiol Ren Physiol. 2008;294(1):F1-F9.

51. Tanner RM, Calhoun DA, Bell EK, et al. Incident ESRD and treatment-resistant hypertension: the reasons for geographic and racial differences in stroke (REGARDS) study. Am J Kidney Dis. 2014;63:781-788.

52. De Nicola L, Borrelli S, Gabbai FB, et al. Burden of resistant hypertension in hypertensive patients with non-dialysis chronic kidney disease. Kidney Blood Press Res. 2011;34(1):58-67.
53. Vollmer WM, Sacks FM, Ard J, et al. Effects of diet and sodium intake on blood pressure: subgroup analysis of the DASH-sodium trial. Ann Intern Med. 2001;135(12):1019-1028.

54. Pimenta E, Gaddam KK, Oparil S, et al. Effects of dietary sodium reduction on blood pressure in subjects with resistant hypertension: results from a randomized trial. Hypertension. 2009;54(3):475-481.

55. Eckel RH, Jakicic JM, Ard JD, et al. 2013 AHA/ACC guideline on lifestyle management to reduce cardiovascular risk: a report of the American College of Cardiology/American Heart Association Task Force on practice guidelines. Circulation. 2014;129:S76-S99.

56. Chobanian AV, Bakris GL, Black HR, et al. Seventh report of the Joint National Committee on Prevention, Detection, Evaluation, and Treatment of High Blood Pressure. Hypertension. 2003;42(6):1206-1252.

57. Mancia G, Fagard R, Narkiewicz K, et al. 2013 ESH/ESC practice guidelines for the management of arterial hypertension. Blood Press. 2014;23:3-16.

58. National Kidney F. K/DOQI clinical practice guidelines for chronic kidney disease: evaluation, classification, and stratification. Am J Kidney Dis. 2002;39(2 suppl 1):S1-S266.

59. Zanchetti A, Mancia G. Longing for clinical excellence: a critical outlook into the NICE recommendations on hypertension management-is nice always good? J Hypertens. 2012;30(4):660-668.

60. Zanchetti A. Hypertension: meta-analyses: first-rank evidence or second-hand information? Nat Rev Cardiol. 2011;8(5):249-251.

61. Messerli FH, Makani H, Benjo A, Romero J, Alviar C, Bangalore S. Antihypertensive efficacy of hydrochlorothiazide as evaluated by ambulatory blood pressure monitoring: a meta-analysis of randomized trials. J Am Coll Cardiol. 2011;57(5):590-600.

62. Roush GC, Holford TR, Guddati AK. Chlorthalidone compared with hydrochlorothiazide in reducing cardiovascular events: systematic review and network meta-analyses. Hypertension. 2012;59(6):1110-1117.

63. Garg JP, Elliott WJ, Folker A, Izhar M, Black HR, Service RUH. Resistant hypertension revisited: a comparison of two universitybased cohorts. Am J Hypertens. 2005;18(5 Pt 1):619-626.

64. Blood Pressure Lowering Treatment Trialists CNinomiya T, Perkovic V, et al. Blood pressure lowering and major cardiovascular events in people with and without chronic kidney disease: metaanalysis of randomised controlled trials. BMJ. 2013;347:f5680.

65. Nishizaka MK, Zaman MA, Calhoun DA. Efficacy of low-dose spironolactone in subjects with resistant hypertension. Am J Hypertens. 2003;16(11 Pt 1):925-930.

66. Chapman N, Dobson J, Wilson S, et al. Effect of spironolactone on blood pressure in subjects with resistant hypertension. Hypertension. 2007;49(4):839-845.

67. Bobrie G, Frank M, Azizi M, et al. Sequential nephron blockade versus sequential renin-angiotensin system blockade in resistant hypertension: a prospective, randomized, open blinded endpoint study. J Hypertens. 2012;30(8):1656-1664.

68. Pisoni R, Acelajado MC, Cartmill FR, et al. Long-term effects of aldosterone blockade in resistant hypertension associated with chronic kidney disease. J Hum Hypertens. 2012;26(8):502-506.

69. Khosla N, Kalaitzidis R, Bakris GL. Predictors of hyperkalemia risk following hypertension control with aldosterone blockade. Am J Nephrol. 2009;30(5):418-424.

70. Eschalier R, McMurray JJ, Swedberg K, et al. Safety and efficacy of eplerenone in patients at high risk for hyperkalemia and/or worsening renal function: analyses of the EMPHASIS-HF study subgroups (Eplerenone in Mild Patients Hospitalization and Survival Study in Heart Failure). J Am Coll Cardiol. 2013;62(17):1585-1593.

71. Chen X, Hassan MO, Jones JV, Sleight P, Floras JS. Baroreflex sensitivity and the blood pressure response to beta-blockade. J Hum Hypertens. 1999;13(3):185-190.

72. Chern CM, Hsu HY, Hu HH, Chen YY, Hsu LC, Chao AC. Effects of atenolol and losartan on baroreflex sensitivity and heart rate variability in uncomplicated essential hypertension. J Cardiovasc Pharmacol. 2006;47(2):169-174. 
73. Lazahari MI, Sattar MA, Abdullah NA, Khan MA, Johns EJ. Clonidine and a novel clonidine analog AL-12 cause sympathoinhibition in spontaneously hypertensive and diabetic spontaneously hypertensive rats. Methods Find Exp Clin Pharmacol. 2008;30(3):193-199.

74. Chan AK, Cheung CW, Chong YK. Alpha-2 agonists in acute pain management. Expert Opin Pharmacother. 2010;11(17):2849-2868.

75. Krum H, Schlaich M, Whitbourn R, et al. Catheter-based renal sympathetic denervation for resistant hypertension: a multicentre safety and proof-of-principle cohort study. Lancet. 2009;373(9671):1275-1281.

76. Smithwick RH, Thompson JE. Splanchnicectomy for essential hypertension; results in 1,266 cases. J Am Med Assoc. 1953;152(16):1501-1504.

77. Rippy MK, Zarins D, Barman NC, Wu A, Duncan KL, Zarins CK. Catheter-based renal sympathetic denervation: chronic preclinical evidence for renal artery safety. Clin Res Cardiol. 2011;100(12): 1095-1101.

78. O'Hagan KP, Thomas GD, Zambraski EJ. Renal denervation decreases blood pressure in DOCA-treated miniature swine with established hypertension. Am J Hypertens. 1990;3(1):62-64.

79. Kassab S, Kato T, Wilkins FC, Chen R, Hall JE, Granger JP. Renal denervation attenuates the sodium retention and hypertension associated with obesity. Hypertension. 1995;25(4 Pt 2):893-897.

80. Symplicity HTN IEsler MD, Krum H, et al. Renal sympathetic denervation in patients with treatment-resistant hypertension (The Symplicity HTN-2 Trial): a randomised controlled trial. Lancet. 2010;376(9756):1903-1909.

81. Esler MD, Krum H, Schlaich M, et al. Renal sympathetic denervation for treatment of drug-resistant hypertension: one-year results from the Symplicity HTN-2 randomized, controlled trial. Circulation. 2012;126(25):2976-2982.
82. Hering D, Mahfoud F, Walton AS, et al. Renal denervation in moderate to severe CKD. J Am Soc Nephrol. 2012;23(7):1250-1257.

83. Kiuchi MG, Maia GL, de Queiroz Carreira MA, et al. Effects of renal denervation with a standard irrigated cardiac ablation catheter on blood pressure and renal function in patients with chronic kidney disease and resistant hypertension. Eur Heart J. 2013;34(28):2114-2121.

84. Howard JP, Nowbar AN, Francis DP. Size of blood pressure reduction from renal denervation: insights from meta-analysis of antihypertensive drug trials of 4,121 patients with focus on trial design: the CONVERGE report. Heart. 2013;99(21):1579-1587.

85. Kandzari DE, Bhatt DL, Sobotka PA, et al. Catheter-based renal denervation for resistant hypertension: rationale and design of the SYMPLICITY HTN-3 Trial. Clin Cardiol. 2012;35(9):528-535.

86. Bhatt DL, Kandzari DE, O'Neill WW, et al. A controlled trial of renal denervation for resistant hypertension. N Engl J Med. 2014;370:13931401.

87. Bisognano JD, Bakris G, Nadim MK, et al. Baroreflex activation therapy lowers blood pressure in patients with resistant hypertension: results from the double-blind, randomized, placebo-controlled Rheos Pivotal Trial. J Am Coll Cardiol. 2011;58(7):765-773.

88. Bakris GL, Nadim MK, Haller H, Lovett EG, Schafer JE, Bisognano JD. Baroreflex activation therapy provides durable benefit in patients with resistant hypertension: results of long-term follow-up in the Rheos Pivotal Trial. J Am Soc Hypertens. 2012;6(2): 152-158.

89. Alnima T, de Leeuw PW, Tan FE, Kroon AA, Rheos Pivotal Trial I. Renal responses to long-term carotid baroreflex activation therapy in patients with drug-resistant hypertension. Hypertension. 2013; 61(6):1334-1339. 